\title{
Discrete Realizations of Contact and Intersection Graphs (Extended Abstract)
}

\author{
Jurek Czyzowicz ${ }^{14}$ (czyzowicz@uqah.uquebec.ca) \\ Evangelos Kranakis ${ }^{24}$ (kranakis@scs.carleton.ca) \\ Danny Krizanc ${ }^{24}$ (krizanc@scs.carleton.ca) \\ Jorge Urrutia $^{34}$ (jorge@csi.uottawa.ca)
}

1 Département d'Informatique, Université du Québec à Hull, Hull, Québec J8X 3X7, Canada.

2 Carleton University, School of Computer Science, Ottawa, ON, K1S 5B6, Canada.

3 University of Ottawa, Department of Computer Science, Ottawa, ON, K1N 9B4, Canada.

${ }^{4}$ Research supported in part by NSERC (National Science and Engineering Research Council of Canada) grant.

\begin{abstract}
Known realizations of geometric representations of graphs, like contact, intersection, etc., are "continuous", in the sense that the geometric objects are drawn in Euclidean space with real numbers as coordinates. In this paper, we initiate the study of dicrete versions of contact and intersection graphs and examine their relation to their continuous counterparts. The classes of graphs arising appear to have interesting properties and are thus interesting in their own right. We also study realizability, characterizations as well as intractability questions for the resulting new classes of graphs.
\end{abstract}

1980 Mathematics Subject Classification: 68R10, 68U05

CR Categories: F.2.2

Key Words and Phrases: Coin, Contact, Intersection, Interval graphs, Discrete, Planar graphs, NP.

\section{Introduction}

Classes of graphs having realizations either as intersection or contact graphs of given geometric objects have attracted the attention of several researchers in the literature, e.g. see the extensive literature cited in $[9,12]$. Problems raised include realization, characterization, as well as intractability. Graphs thus realized have interesting applications ranging from scheduling theory [9] to motion planning $[1]$.

Known studies of such graph-classes have only been concerned with "continuous" realizations of the concepts of "intersection" and "contact", in which the contact or intersection points have real numbers as coordinates. However, practical realizations of these concepts would seem to require the more natural concepts of discrete "intersection" and "contact" points, in the sense that the 
contact or intersection points have rationals or even "integers with a restricted range" as coordinates. By this we mean that the geometric objects have a predetermined set of points and only these points can serve as contact or intersection points among the objects.

A coin is a closed circular disc in the plane. Coin graphs are constructed from discs with disjoint interiors. Vertices of the graph are the coins. Two coins are adjacent if they touch each other. Such graphs are planar and have been considered previously in the literature [12]. A remarkable theorem attributed to P. Koebe by H. Sachs [12] states that every finite simple planar graph is a coin graph. The most recent proof of this theorem is given in [4]. It is intersesting to note that the discs required in the above representation of the planar graph are of arbitrary size. In addition, a related result in [7] shows that planar graphs are precisely the contact graphs of isosceles triangles.

Another class of graphs we will study are discrete intersection graphs. Among these are interval and unit square graphs which have been studied extensively in the literature [9]. Linear recognition algorithms for these graphs have appeared in $[9,5,6]$, etc.

\subsection{Preliminaries and notation}

The class of coin graphs considered here are constructed from a single size coin.

Definition 1. The class of coin graphs, denoted by $\mathcal{C}$, is defined as follows. Vertices are represented by unit discs such that the interiors of any pair of such discs have empty intersection. Two discs are adjacent if they touch.

Clearly the graphs in $\mathcal{C}$ are planar. The problem of recognizing whether a given graph is in the class $\mathcal{C}$ has been shown recently to be NP-complete [3].

Another class of graphs we will consider in this paper is the class of unit interval graphs (all intervals considered in the sequel are closed).

Definition 2. The class of unit interval graphs, denoted by $\mathcal{I}$, is defined as follows. Vertices are represented by unit intervals. Two intervals are adjacent if they have a nonempty intersection.

It is known that unit interval graphs are precisely the interval graphs containing no induced copy of $K_{1,3}$ [9]. Thus the discrete unit interval graphs are a refinement of the unit interval graphs.

Notation.

From now on and for the rest of the paper if $\mathcal{G}$ denotes a class of graphs then $\mathcal{G}^{n}$ denotes the set of graphs in $\mathcal{G}$ with exactly $n$ vertices. For example, we have the classes $\mathcal{I}^{n}, \mathcal{C}^{n}$, etc.

\subsection{Results of the paper}

In this paper we initiate the study of dicrete versions of the above mentioned classes of graphs. The classes of graphs arising appear to have interesting properties and are thus interesting in their own right. We also study realizability, characterizations as well as intractability questions for the resulting new graphs. 


\section{Discrete Coin Graphs}

In the sequel we define new classes of planar coin graphs. For each integer $k \geq 3$ consider a regular $k$-gon $P_{k}$.

Definition 3. The class of polygonal coin graphs, denoted by $\mathcal{P}_{k}$ is defined as follows. Vertices are represented by isothets of the regular $k$-gon $P_{k}$ such that the interiors of any pair of such $k$-gons have empty intersection. Two $k$-gons are adjacent if they touch at a polygon vertex.

Clearly the graphs in the class $\mathcal{P}_{k}$ are planar. We can prove the following theorem.
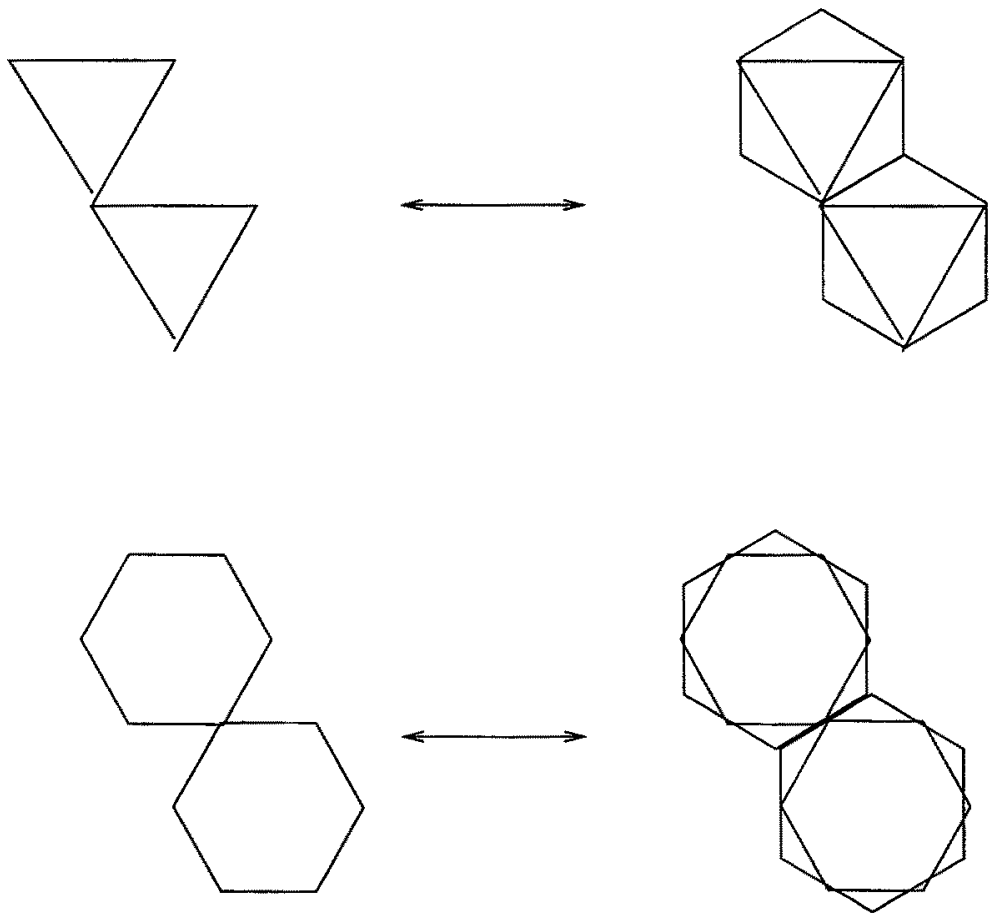

Fig. 1. The polygonal transformations required to prove that all polygonal coin graphs are coin graphs, for the case of equilateral triangles and hexagons.

Theorem 4. All polygonal coin graphs are coin graphs, i.e. $\mathcal{P}_{k} \subseteq \mathcal{C}$, for all integers $k$.

Proof (OUtuine) of Theorem 4. The theorem is easy for $k$ even. Consider the layout of regular $k$-gons representing a given graph. Inscribe each polygon 
in a disc. Since $k$ is even the resulting discs have mutually disjoint interiors. Moreover, the contact graph realized by these discs is identical to the contact graph realized by the $k$-gons. (See also Figure 1 .) Therefore, we need only prove the assertion $\mathcal{P}_{k} \subseteq \mathcal{C}$ in the case $k$ is odd.

In the sequel it will be convenient to use the notation $\mathcal{P}_{k}^{v e}$ as identical to the notation $\mathcal{P}_{k}$ in order to indicate that adjacency is determined by polygons touching on a plygon vertex. It will also be convenient to consider "adjacency" for polygonal layouts in which two $k$-gons are adjacent if they touch at a whole edge. More precisely we have.

Definition 5. The class $\mathcal{P}_{k}^{\text {ed }}$ is defined as follows. Vertices are represented by isothets of the regular $k$-gon $P_{k}$ such that the interiors of any pair of such $k$ gons have empty intersection. Two $k$-polygons are adjacent if two polygon edges match.

Thus, by definition we have $\mathcal{P}_{k}^{e d}=\emptyset$, if $k$ is odd. We can prove the following lemma which easily implies Theorem 4.

Lemma 6. For each integer $k \geq 3$ we have that

1. if $k$ is even then $\mathcal{P}_{k}^{v e} \subseteq \mathcal{P}_{k l}^{v e}$, for all integers $l$,

2. if $k$ is odd then $\mathcal{P}_{k}^{v e}=\mathcal{P}_{2 k}^{e d}$,

3. if $k$ is even then $\mathcal{P}_{k}^{v e}=\mathcal{P}_{k}^{\text {ed }}$.

Proof of Lemma 6 Consider a side of the $k$-gon $P_{k}$. The side adjacent to it forms an angle $2 \pi / k$ with it (see Figure 2). Let $a, b$ be the exterior angles formed by two $k$-gons intersecting at a vertex. It is clear that $a+b=4 \pi / k$. If follows easily from angle considerations of the corresponding $k$-gons that if $k$ is even then $a=b=2 \pi / k$, while if $k$ is odd then one of the angles is $\pi / k$ while the other is $3 \pi / k$.
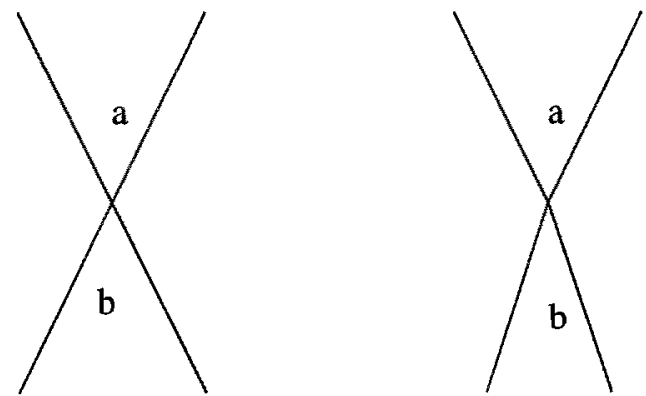

Fig. 2. The angles $a, b$ of two touching polygons. In the picture to the left $k$ is even, while in the picture to the right $k$ is odd.

Now we can give the proof of the lemma. We transform the given polygon $P_{k}$ into a new polygon $Q_{k}$. For given $l$, we inscribe $P_{k}$ into a regular $k l$-gon $Q_{k l}$ 
such that the vertices of $P_{k}$ are also vertices of $Q_{k l}$. Now suppose that $P_{k}$ and $P_{k}^{\prime}$ are two touching $k$-gons. We consider separately the case where $k$ is even and $k$ is odd. It is easy to show that if $k$ is even then the $k l$-gons $Q_{k l}$ and $Q_{k l}^{\prime}$ touch at a vertex if and only if the $k$-gons $P_{k}$ and $P_{k}^{\prime}$ touch at a vertex. This implies that if $k$ is even then $\mathcal{P}_{k}^{v e} \subseteq \mathcal{P}_{k l}^{v e}$, for all integers $l$. If $k$ is odd and the polygons $P_{k}$ and $P_{k}^{\prime}$ touch at a vertex then the previous observations imply that one of the exterior angles at the contact point of the polygons is $\pi / k$ while the other is $3 \pi / k$. It follows that the corresponding polygons $Q_{k l}$ and $Q_{k l}^{\prime}$ have nontrivial intersection. Moreover, this intersection is a polygon edge exactly when $l=2$. It follows that if $k$ is odd then $\mathcal{P}_{k}^{v e} \subseteq \mathcal{P}_{2 k}^{e d}$.

To prove that $\mathcal{P}_{2 k}^{e d} \subseteq \mathcal{P}_{k}^{v e}$ we essentially reverse the above argument. For a given $2 k$-gon $P_{2 k}$ inscribe a $k$-gon as follows: form the $k$-gon $Q_{k}$ with vertices the mid-points of the edges of $P_{2 k}$. It follows that two $2 k$-gons $P_{2 k}$ and $P_{2 k}^{\prime}$ touch at an edge if and only if the $k$-gons $Q_{k}$ and $Q_{k}^{\prime}$ touch at a vertex. Which completes the proof of the assertion.

The assertion $\mathcal{P}_{k}^{u e}=\mathcal{P}_{k}^{e d}$ for $k$ even follows essentially the same ideas. One inscribes the given $k$-gon $P_{k}$ into a $k$-gon $Q_{k}$ such that the vertices of $P_{k}$ are midpoints of edges of $Q_{k}$, and vice versa. Details of the proof are left to the reader.

This completes the proof of Lemma 6 and hence also the proof of Theorem 4.

This raises the following interesting problem.

Problem 7. Is there a constant $c$ such that $\mathcal{P}_{k}=\mathcal{C}$, for all $k \geq c$ ?

We show this is impossible by proving the following theorem.

Theorem 8. For each $k$, there exist an $n$-vertex unit-disc contact graph with $n \in O(k)$ which is not representable by regular $k$-gons.

Proof (OutLine) We consider the contact graph depicted in Figure 3. It consists of the following components. An internal circle $C$, straight line double chains $L$ around the internal circle and at equal angles, and an external circle. Both internal and external circles consist of unit circles. The external circle consists of circular chain segments $S$. These parts are connected in such a way that an imobile structure is formed. The corresponding parts can be constructed with unit discs thus forming a contact disc graph.

Let successive segments of type $L$ be such that they are forming an angle of size $2 \pi / n$. It is clear that we can achieve this by using a total of $O(n)$ unit discs in the graph. If $k \notin \Omega(n)$ then we note by using only regular $k$-gobs it will be impossible to form the graph depicted in Figure 3.

\subsection{Complexity of Recognition Problem}

Theorem 9. For each $k$, the problem of recognizing whether a given graph is in the class $\mathcal{P}_{4 k}$ is NP-complete. 


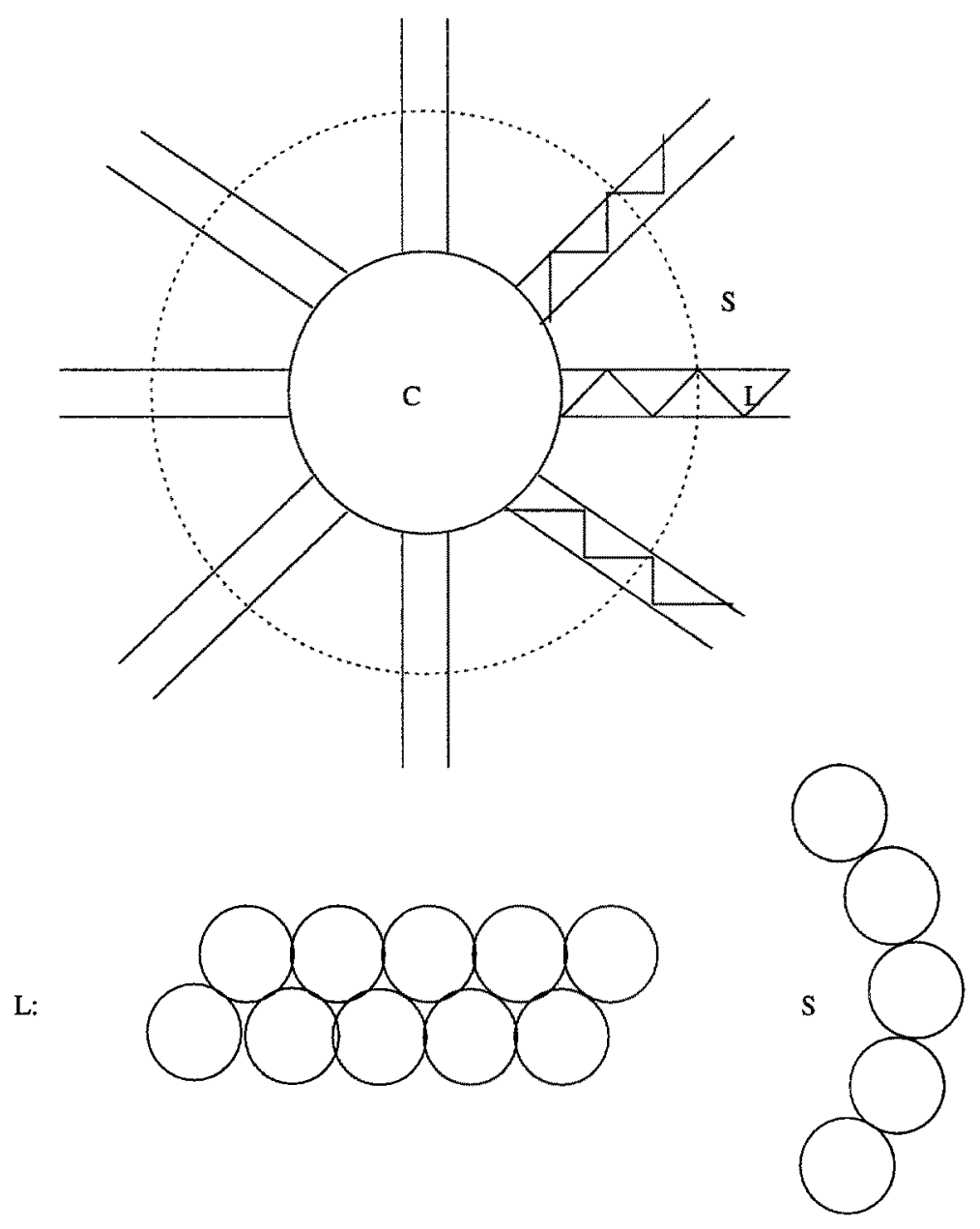

Fig. 3. Proving the lower bound in discretizing disc graphs. The graph is constructed from the components $L, S$ and the circle $C$ which is formed of a circular chain of unit discs.

Proof (OUTLINe) We give only the argument for the class $\mathcal{P}_{4}$. Extensions to the classes $\mathcal{P}_{4 k}$ are straightforward and will be given in the full paper.

We show how to reduce the problem of laying out a graph on a grid with dilation one to the above graph recognition problem. The former problem is known to be NP-complete $[2,11]$ even for the case when the graph is restricted to be a tree. For a given graph $G=(V, E)$ we define a new graph $G^{\prime}=\left(V^{\prime}, E^{\prime}\right)$ such that

$$
G^{\prime} \in \mathcal{P}_{4} \Leftrightarrow G \text { is embedable on a grid with dilation } 1 \text {. }
$$


It follows that the recognition problem for the class $\mathcal{P}_{4}$ is NP-complete. Since $G$ is a tree if and only if $G^{\prime}$ is a tree it will also follow that the problem of recognizing whether a given tree is in the class $\mathcal{P}_{4}$ is NP-complete.

The graph $G^{\prime}$ is defined from $G$ as follows. We replace each edge $\{u, v\}$ with the following caterpillar $C(u, v)$

- vertices: $u, v, u_{1}, u_{2}, u_{3}, u_{4}, u_{5}$ and $x_{1}, x_{2}, y_{1}, y_{2}$.

- edges:

$$
\begin{gathered}
\left\{u, u_{1}\right\},\left\{u_{1}, u_{2}\right\},\left\{u_{2}, u_{3}\right\},\left\{u_{3}, u_{4}\right\},\left\{u_{4}, u_{5}\right\},\left\{u_{5}, v\right\}, \text { and } \\
\left\{x_{1}, u_{2}\right\},\left\{x_{2}, u_{4}\right\},\left\{y_{1}, u_{2}\right\},\left\{y_{2}, u_{4}\right\} .
\end{gathered}
$$

The caterpillar itself is depicted in Figure 4. To prove the equivalence (1) observe

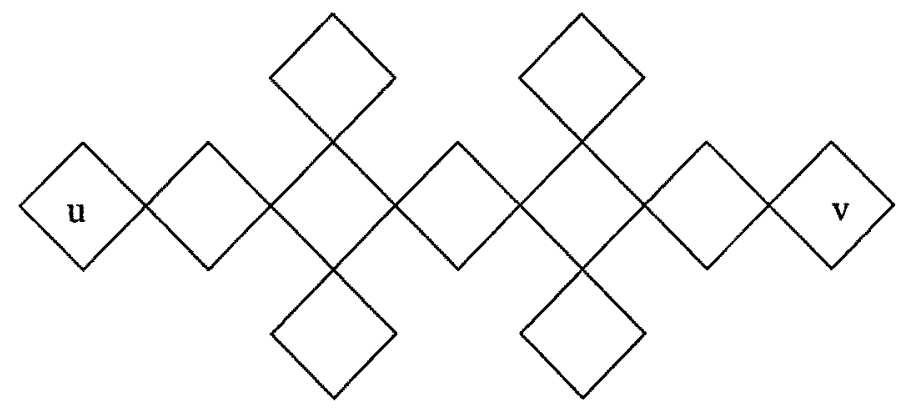

Fig. 4. The caterpillar $C(u, v)$ replacing the edge $\{u, v\}$.

that if the caterpillar $C(u, v)$ is represented in $\mathcal{P}_{4}$ then the centers of the squares representing the verices $u, v$ must both lie either on a vertical or horizontal line. This completes the proof of the theorem.

An interesting problem is the following.

Problem 10. For each $k$, is the problem of recognizing whether a given graph is in the class $\mathcal{P}_{k}$, NP-complete?

\section{Discrete Intersection Graphs}

Interval and unit square graphs have been extensively studied in the literature [9]. Linear recognition algorithms for these graphs have appeared in $[9,5,6]$, etc. In the sequel we define new classes of unit interval graphs. 


\subsection{Discretizing intersection graphs}

Intersection graphs of convex objects can be discretized by replacing intersection points with rational numbers which are sufficiently close to the original intersection points. To illustrate this point the following argument which is stated for the classes of intersection graphs of unit squares can be used for intersection graph representation by any type of convex objects.

Theorem 11. Intersection graphs of unit squares can be realized as intersection graphs of unit squares all of whose corners have rational endpoints.

Proof We consider the Manhattan norm for points in the plane, $|(x, y)|=x+y$. Let $\left\{S_{u}: u \in V\right\}$ be a collection of closed unit squares representing the graph $G=(V, E)$, i.e. for all vertices $u, v$,

$$
E(u, v) \Leftrightarrow S_{u} \cap S_{v} \neq \emptyset .
$$

Let $c_{u}$ be the center of the square $I_{u}$. Clearly, there exists a real $r>0$ such that for all $u, v$

$$
\left|c_{u}-c_{v}\right|<1 \Rightarrow\left|c_{u}-c_{v}\right|<1-r \text {, and }\left|c_{u}-c_{v}\right|>1 \Rightarrow\left|c_{u}-c_{v}\right|>1+r .
$$

Choose a sequence $\left\{r_{u}: u \in V\right\}$ of pairs of reals such that for all $u, v$,

$-\left|r_{u}\right|<r / 2$,

$-c_{u}+r_{u}$ has both components rational, and

- if $c_{u}-c_{v}$ has both components integers then $r_{u}=r_{v}$.

Now consider the unit squares $T_{u}$ with center $c_{u}+r_{u}$. It is easy to verify that this sequence of squares realizes the same graph $G$. Since all the corners of the squares are rationals it is starightforward that we can find an integer $k$ such that $\mathcal{S}^{n}=\mathcal{S}_{k}^{n}$.

One of the problems to be studied in this paper is the following.

Problem 12. What is the complexity of a discrete realization of a geometric graph when corresponding points are only allowed to have integer coordinates with "limited range"?

\subsection{Discrete unit interval graphs}

Definition 13. Let $k$ be a given integer. To define the class of $k$-discrete unit interval graphs we consider intervals of identical size over the integers. A graph belongs to the class $\mathcal{I}_{k}$ if it can be represented by a collection of pairwise distinct intervals over the integers such that each interval has length exactly $k$ and such that interval intersections can only occur at integer points.

Since all intervals are of equal length it is clear that $\mathcal{I}_{k} \subseteq \mathcal{I}$, for all $k$.

Theorem 14. For all $n$ there exists an integer $k \leq 2^{n-1}$ such that $\mathcal{I}^{n}=\mathcal{I}_{k}^{n}$. 
Proof Let $\left\{I_{u}: u \in V\right\}$ be a collection of closed unit intervals representing the graph $G=(V, E)$, i.e. for all vertices $u, v, E(u, v) \Leftrightarrow I_{u} \cap I_{v} \neq \emptyset$.

We prove the existence of a unit interval realization of the graph such that no two endpoints of intervals of the new realization are identical.

Let $l(I), r(I)$ denote the left and right endpoints of the interval $I$. We prove the existence of a unit interval representation $\left\{J_{u}: u \in V\right\}$ of the graph in such a way that for all $u, v$,

$-l\left(I_{u}\right)<l\left(I_{v}\right) \Leftrightarrow l\left(J_{u}\right)<l\left(J_{v}\right)$

$-\left|\left\{l\left(J_{u}\right), r\left(J_{u}\right): u \in V\right\}\right|=2 n$,

- for any $x \neq y$ in the set $\left\{l\left(J_{u}\right), r\left(J_{u}\right): u \in V\right\}$ we have that $|x-y| \geq 1$.

The proof is by induction on $n$. The inductive step $n=1$ is trivial. Assume the induction hypothesis is true for interval graphs with $n-1$ vertices. Remove the interval with the smallest left endpoint, say $I_{a}$. Consider all intervals $\left\{I_{u} ; u \neq a\right\}$ intersecting $I_{a}$. Since all intervals have equal lengths, it is easy to show that the set $\left\{I_{u}: u \neq a, I_{u} \cap I_{a} \neq \emptyset\right\}$ forms a clique. By the induction hypothesis the set $\left\{J_{u}: u \neq a, I_{u} \cap I_{a} \neq \emptyset\right\}$ also forms a clique. In particular their left endpoints must lie within an interval of length $2^{n-2}$. Since by the induction hypothesis no two endpoints can coincide, in follows that these left endpoints must lie within an interval of length $2^{n-2}-1$. Let $r$ be the rightmost left endpoint among the intervals $\left\{J_{u}: u \neq a, I_{u} \cap I_{a} \neq \emptyset\right\}$. Let $J_{a}$ be the closed interval

$$
J_{a}=\left[r-2^{n-2}+\frac{1}{2}, r+\frac{1}{2}\right] .
$$

For each $u$ define the intervals $\bar{J}_{u}=\left[2 l\left(J_{u}\right), 2 r\left(J_{u}\right)\right]$. It is clear that each interval has length $2^{n-1}$. Moreover, it is easy to see that the resulting sequence $\left\{\bar{J}_{u}: u \in\right.$ $V\}$ of intervals represents the same graph $G$ and satisfies all the conditions of the inductive steps. This proves the theorem.

It is interesting to note that Theorem 14 improves on Theorem 3.2 of [5] which implies that for all $n$ there exists an integer $k \leq n$ ! such that $\mathcal{I}^{n}=\mathcal{I}_{k}^{n}$.

Theorem 15. There exist graphs $G \in \mathcal{I}_{n / 2-1}^{n} \backslash \mathcal{I}_{n / 2-2}^{n}$, for $n \geq 2$.

Proof Let $n=2 m$. Consider the unit interval graph with vertex set $V=$ $\left\{u_{1}, \ldots, u_{m}, v_{1}, \ldots, v_{m}\right\}$. Edges are defined as follows. Vertex $u_{i}$ is adjacent exactly to the vertices

$$
u_{1}, u_{2}, \ldots, u_{m}, v_{1}, v_{2}, \ldots, v_{i-1}
$$

and vertex $v_{i}$ is adjacent exactly to the vertices

$$
v_{1}, v_{2}, \ldots, v_{m}, u_{i+1}, v_{i+2}, \ldots, u_{m}
$$

Since the degree sequence of the graph attains $m$ different values it is easy to see that $G \notin \mathcal{I}_{n / 2-2}^{n}$. The rest of the statement of the theorem is immediate. 
Definition 16. An interesting parameter for a (unit) interval graph $G$ is $\mathcal{I}(G)$ which is the smallest integer $m$ such that a discrete unit interval representation of the graph can be drawn with all integers of all interval endpoints in the range $[0, m]$.

Theorem 14 implies that for any unit interval graph $G, \mathcal{I}(G) \leq n 2^{n-1}$. We can also prove the following lower bound.

Theorem 17. There exist graphs $G \in \mathcal{I}^{n}$ such that $\mathcal{I}(G) \in \Omega(n \log n)$.

Proof (Outline) Let $i(n)$ be the number of unit interval graphs on $n$ vertices. Assume we could draw all unit interval graphs within the integer range $[1, m]$ with all (pairwise distinct) intervals of length exactly $k$. Since unit intervals are uniquely determined by a unique point (say, their cenrter) it is clear that there exist at most $\left(\begin{array}{c}m \\ n\end{array}\right)$ such graphs. Hence, $i(n) \leq\left(\begin{array}{l}m \\ n\end{array}\right)$. This implies that $n ! \cdot i(n) \leq m^{n}$. Trivial calculations show that this implies that $m \in \Omega(n \log n)$.

An interesting problem is the following.

Problem 18. Give an algorithm for recognizing membership in the class $\mathcal{I}_{k}$. Is this an NP-complete problem? A related question is the following. For each graph $G \in \mathcal{I}^{n}$ compute $\operatorname{disc}_{\mathcal{I}}(G)=\min _{k}\left\{G \in \mathcal{I}_{k}^{n}\right\}$. A similar question is the following. For each graph $G \in \mathcal{I}^{n}$ compute $\mathcal{I}(G)$. Can we tighten the bounds $c n \log n \leq \mathcal{I}(G) \leq n 2^{n-1}$ ?

\subsection{Discrete unit square intersection graphs}

Definition 19. The class of unit square graphs, denoted by $\mathcal{S}$, is defined as follows. Vertices are represented by unit squares. Two vertices are adjacent if they have a nonempty intersection.

Definition 20. Let $k$ be a given integer. To define the class of $k$-discrete unit square graphs we consider squares of identical side over the integer square lattice of integers. A graph belongs to the class $\mathcal{S}_{k}$ if it can be represented by a collection of squares over the square lattice of integers such that the side of each square is an interval of length exactly $k$.

Since all squares are of equal length it is clear that $\mathcal{S}_{k} \subseteq \mathcal{S}$, for all $k$. We can ask similar questions as with unit interval graphs.

Theorem 21. For all $n$ there exists an integer $k \leq 2^{n-1}$ such that $\mathcal{S}^{n}=\mathcal{S}_{k}^{n}$.

Proof (Outline) Consider the two unit interval graphs formed by projecting the squares representing a given graph on the $x$ and $y$ axis. Then use the result of Theorem 14.

Problem 22. Give an algorithm for recognizing membership in the class $\mathcal{S}_{k}^{n}$. Is this an NP-complete problem? A related question is the following. For each graph $G \in \mathcal{S}^{n}$ compute $\operatorname{disc}_{\mathcal{S}}(G)=\min _{k}\left\{G \in \mathcal{S}_{k}^{n}\right\}$. 
We also state without proof the following two theorems.

Theorem 23. For each graph $G \in \mathcal{S}$ let $G_{x}, G_{y}$ be the unit interval graphs obtained by projecting the squares representing $G$ on the $x$ - and $y$-axis. Then $\max \left\{\operatorname{disc}_{\mathcal{I}}\left(G_{x}\right), \operatorname{disc}_{\mathcal{I}}\left(G_{y}\right)\right\} \leq \operatorname{disc}_{\mathcal{S}}(G) \leq \operatorname{disc}_{\mathcal{I}}\left(G_{x}\right) \cdot \operatorname{disc}_{\mathcal{I}}\left(G_{y}\right)$.

Theorem 24. If $G_{1}, G_{2} \in \mathcal{I}^{n}$ such that $\operatorname{disc}_{\mathcal{I}}\left(G_{1}\right)=\operatorname{disc}_{\mathcal{I}}\left(G_{2}\right)=k$ then there exists a graph $G \in \mathcal{S}^{n}$ such that $G_{x}=G_{1}, G_{y}=G_{2}$ and $\operatorname{disc}_{\mathcal{S}}(G)=k$.

\section{Conclusion}

In this paper we considered discrete realizations of contact and intersection graphs. Contact graphs of regular polygons form a hierarchy of planar graphs and the recognition problem for such graphs is NP-hard. In contrast to this, intersection graphs are always descretizable and bounds on the complexity of the resulting discetizations are given.

\section{Acknowledgements}

Many thanks to Prosenjit Bose for useful converations that improved the presentation.

\section{References}

1. S. Abramowski, B. Lang, and H. Miller, "Moving Regular k-gons in Contact", In proceedings of International Workshop WG'88 on Graph-Theoretic Concepts in Computer Science, Jan van Leewen, editor, Springer Verlag Lecture Notes in Computer Science, vol. 344, pp. 229-242, 1989.

2. S. N. Bhatt and S. S. Cosmadakis, "The Complexity of Minimizing Wire Lengths in VLSI Layouts", Information Processing Letters 25 (1987) 263-267.

3. H. Breu and D. G. Kirkpatrick, "On the Complexity of Recognizing Intersection and Touching Graphs of Disks", In proceedings of Graph Drawing 95, pp. 88-98. Springer Verlag Lecture Notes in Computer Science.

4. G. R. Brightwell, and E. R. Scheinerman, "Representation of Planar Graphs", SIAM Journal on ???, Vol. 6, No. 2, pp. 214 - 229, May 1993.

5. D. G. Corneil, H. Kim, S. Natarajan, S. Olariu, and A. P. Sprague, "Simple Linear Time Recognition of Unit Interval Graphs", Information Processing Letters 55(1995) 99-104.

6. C. M. Herrera de Figueiredo, J. Meidanis, C. Picinin de Mello, "A Linear-Time Alforithm for Proper Interval Graph Recognition", Information Processing Letters 56 (1995) 179-184.

7. H. de Fraysseix, P. Ossona de Mendez, and P. Rosenstiehl, "On 'Triangle Contact Graphs", Combinatorics, Probability and Computing (1994) 3, 233 - 246.

8. D. R. Fulkerson and O. A. Gross, "Incidence Matrices and Interval Graphs", Pacific Journal of Mathematics, 15, 835-855, 1985.

9. M. C. Golumbic, "Algorithmic Graph Theory and Perfect Graphs", Academic Press, New York, 1980. 
10. N. Korte and R. H. Möhring, "A Simple Linear-Time Algorithm to Recognize Interval Graphs", In proceedings of International Workshop WG'86 on GraphTheoretic Concepts in Computer Science, G. Tinhofer and G. Schmidt, editors, Springer Verlag Lecture Notes in Computer Science, vol. 246, 1987.

11. Z. Miller and J. B. Orlin, "NP-Completeness for Minimizing Maximum Edge Length in Grid Embeddings", Journal of Algorithms 6, 10-16 (1985).

12. H. Sachs, "Coin Graphs, Polyhedra, and Conformal Mapping", Discrete Mathematics 134 (1994) 133-138. 